\title{
Water-Jet Cavitation Shock Bulging as Novel Microforming Technique
}

\author{
Fuzhu Li, Haiyang Fan, Yuqin Guo, Zhipeng Chen, Xu Wang, Ruitao Li, Hong Liu and Yun Wang*(0)
}

\begin{abstract}
With the continuous expansion of the application range of microelectromechanical systems, microdevice forming technology has achieved remarkable results. However, it is challenging to develop new microforming processes that are low cost, environmentally friendly, and highly flexible; the high-energy shock wave in a cavitation bubble's collapse process is used as the loading force. Herein, a new process for the microbulging of the water-jet cavitation is proposed. A series of experiments involving the water-jet cavitation shock microbulging process for TA2 titanium foil is performed on an experimental system. The microforming feasibility of the water-jet cavitation is investigated by characterizing the shape of the formed part. Subsequently, the effects of the main parameters of the water-jet cavitation on the bulging profile, forming depth, surface roughness, and bulging thickness distribution of TA2 titanium foil are revealed. The results show that the plastic deformation increases nonlinearly with the incident pressure. When the incident pressure is $20 \mathrm{MPa}$, the maximum deformation exceeds $240 \mu \mathrm{m}$, and the thickness thinning ratio changes within $10 \%$. The microbulging feasibility of water-jet cavitation is verified by this phenomenon.
\end{abstract}

Keywords: Water-jet cavitation, Microbulging, TA2 titanium foil, Shock forming, Plastic deformation

\section{Introduction}

The science and technology of microdevice forming has received significant attention and hence in-depth research has been performed by scholars. However, the traditional microforming process is accompanied by a series of insurmountable problems owing to microscale effects [1-3]. Additionally, the existing deep reactive ion etching, photolithography, lithography, electrochemistry, micro electric discharge machining (micro-EDM), laser shock, and other microprocessing technologies exhibit problems such as low efficiency, easy pollution, complex process flow, large investment, and difficult operation [4, 5]. Therefore, new microforming processes are necessitated for solving the current problems in microforming.

A water-jet cavitation carrying numerous cavitation bubbles is formed after a high-pressure water jet passes through the nozzle. The cavitation bubbles collapse near

\footnotetext{
*Correspondence: wangyun@ujs.edu.cn

School of Mechanical Engineering, Jiangsu University, Zhenjiang 212013, China
}

the solid wall, and high energy is produced under complex conditions such as confining pressure and strong shear $[6,7]$. Water-jet cavitation has been widely used in cleaning, sewage treatment, and rock mining [8-10]. The application of water-jet cavitation to the surface modification of materials has become a popular topic in recent years. Soyama et al. [11] conducted cavitation shot peening research on copper, aluminum, stainless steel, and other materials. The effects of the nozzle structure, spray pressure, target distance, and other process parameters on the microstructure changes of the materials were revealed. Marcon et al. [12] used the high-energy shock wave of water-jet cavitation to bend the duralumin alloy sheet of an aircraft wing and discovered that the cavitation nozzle exhibited a large deformation curvature. Tsuda et al. [13] combined quenching with cavitation shot peening to strengthen a carbon-steel spiral gear. A $100-\mu \mathrm{m}$-deep strengthening layer and a residual compressive stress of $600 \mathrm{MPa}$ was formed. Soyama et al. [14, 15] were pioneers in determining the feasibility of sheet metal forming through water-jet cavitation. Their results 
showed that the sheet metal underwent high-speed plastic deformation and satisfactory plastic deformation as the cavitation bubbles collapsed. However, reports regarding the application of water-jet cavitation to foil microforming are scarce.

High-energy shock waves produced by the bubble collapse of a cavitation water jet were used as the loading force based on previous microplastic forming theory in this study. An experiment was performed on a TA2 titanium foil with a thickness of $100 \mu \mathrm{m}$ to analyze the effects of cavitation water jet process parameters on the microplastic forming of TA2 titanium foil. First, the new technology of cavitation water jet micro-forming was investigated herein, where the results provide new insights for microdevice formation.

\section{Methodology}

\subsection{Experimental Setup}

Figure 1 shows the experimental setup of the water-jet cavitation shock microbulging process. The experiments were performed in a transparent water tank filled with tap water at $25 \pm 2{ }^{\circ} \mathrm{C}$. According to Refs. [16-18], the cavitation nozzle with a $d / L$ ratio of $1: 8$, as shown in Figure 2, was designed to generate a periodic cavitating jet. Hence, when the throat diameter of the nozzle $d$ was 1.5 $\mathrm{mm}$, its throat length $L$ was $12 \mathrm{~mm}$, and the expansion angle $\theta$ was set as $30^{\circ}$. Subsequently, the cavitation nozzle was mounted on a supporting device and adjusted by a standoff distance $S$, i.e., the distance between the nozzle outlet and workpiece surface. In this experimental setup, the water flow through the cavitation nozzle was driven by a plunger pump to generate a submerged cavitating jet in the test cell.

It is noteworthy that the entire forming device was fixed on a platform located in a water-filled transparent water tank. The standoff distance was set as 120

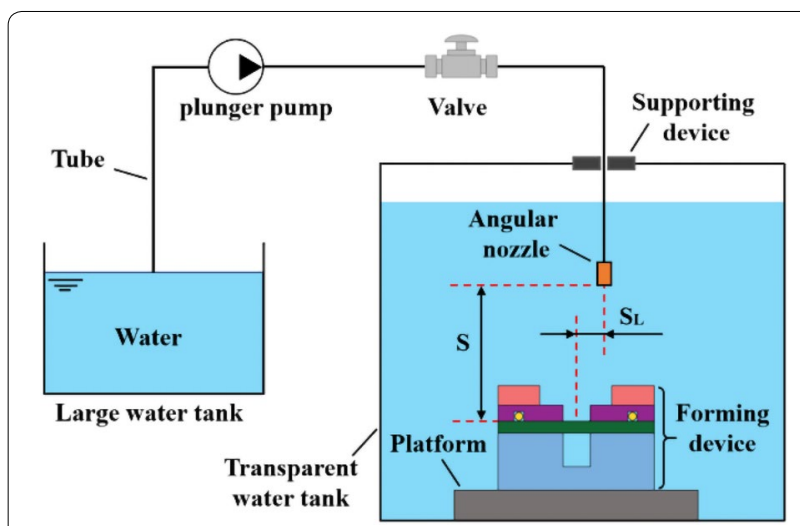

Figure 1 Experimental setup of water-jet cavitation shock microbulging process

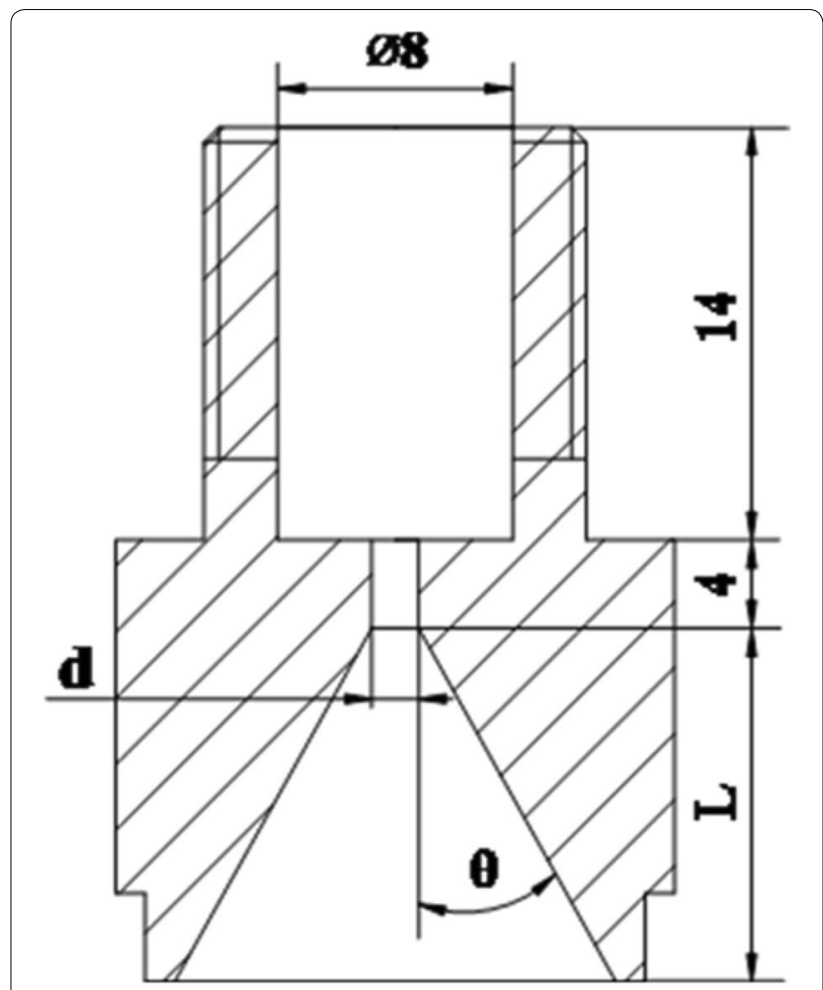

Figure 2 Geometry and structure of cavitation nozzle

mm through trial and error based on a previous study [18]. It is important to achieve a sufficient cavitating jet intensity that can induce a concentrated collapse in cavitation bubbles without any pitting or damages due to overformation.

Inside the test cell, the forming device should be perpendicular to the cavitating nozzle, and the incident pressure applied on the surface of the foil is indicated by a pressure gauge installed on the plunger pump. According to the preliminary experiment results, as shown in Figure 3, an impact zone with good forming effects under different incident pressures appeared between two concentric circles whose radii were $R_{1}$ and $R_{2}$, and whose center was aligned with the jet axis. Considering the region between the two concentric circles with radii $R_{1}=12.3 \mathrm{~mm}$ (i.e., the maximum value of $R_{1}$ ) and $R_{2}=19.3 \mathrm{~mm}$ (i.e., the minimum value of $R_{2}$ ) was always a stable impact zone in the range of incident pressure investigated (i.e., 8, 12, 16, and $20 \mathrm{MPa}$ ), a compromise value of $14 \mathrm{~mm}$ was set for the axial spacing $S_{L}$ (i.e., the distance between the centerline of the micromold cavity and the jet axis) to ensure that the cavitation impact forming zone was located stably in the central region of the micromold.

According to Ref. [19], as a measure of the resistance of the flow to cavitation, the cavitation number $\sigma$ is 


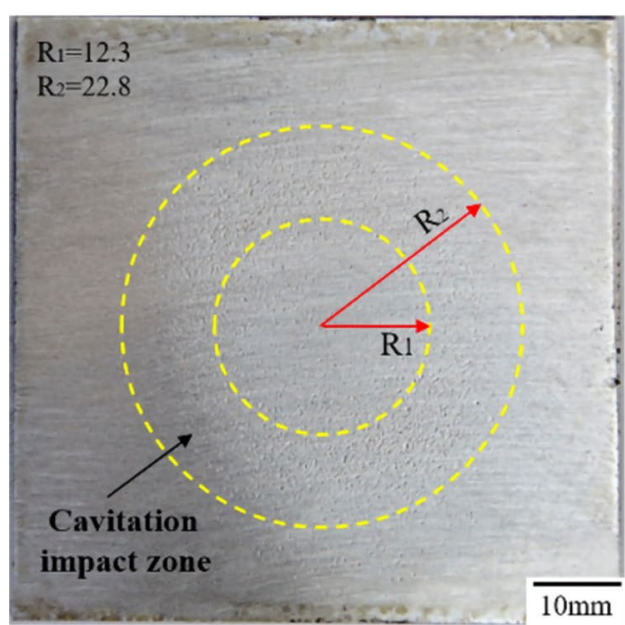

a $8 \mathrm{MPa}$

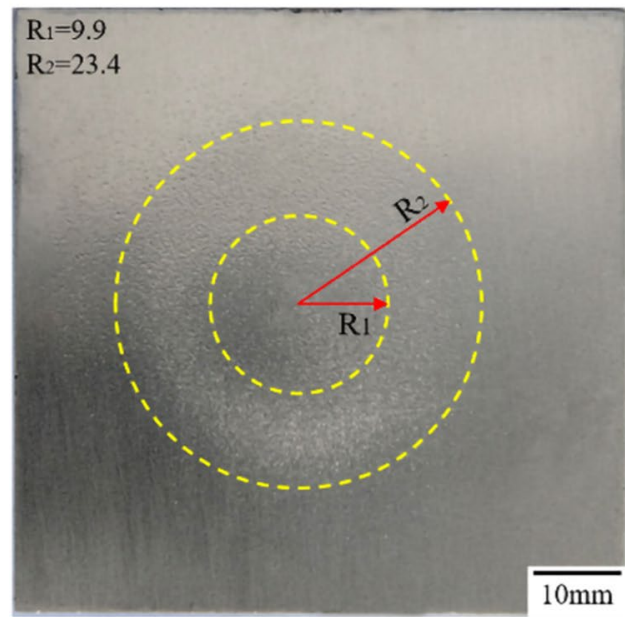

c $16 \mathrm{MPa}$

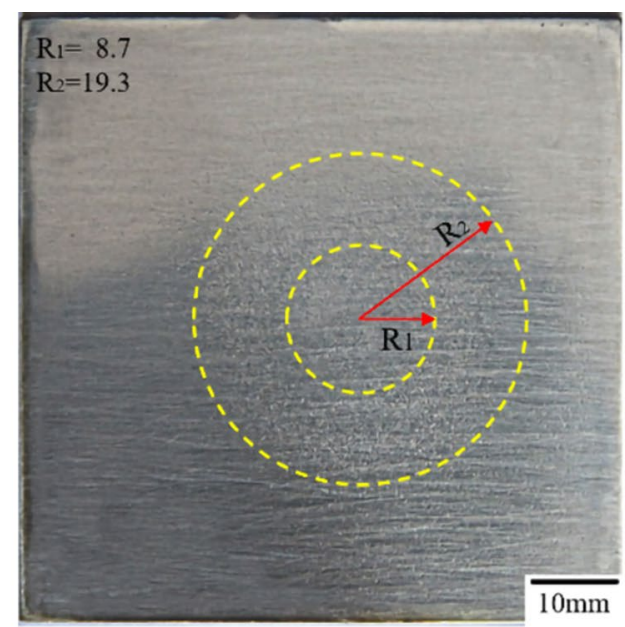

b $12 \mathrm{MPa}$

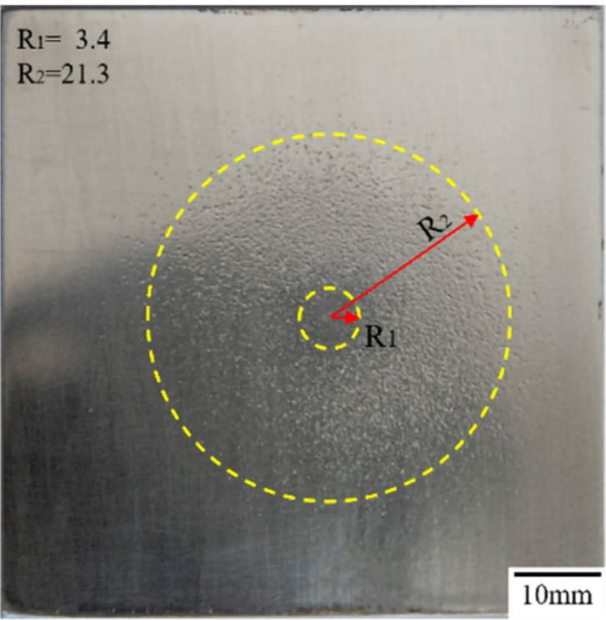

d $20 \mathrm{MPa}$

Figure 3 Impact zone of cavitation water jet under different incident pressures

defined as the ratio of the incident (upstream) pressure $P_{1}$ to the downstream pressure $P_{2}$, as shown in Eq. (1):

$$
\sigma=\frac{P_{2}}{P_{1}},
$$

where the incident pressure $P_{1}$ is one of the key parameters in the water-jet cavitation shock microbulging process and was set as $8,12,16$, and $20 \mathrm{MPa}$. The downstream pressure $P_{2}$ was maintained at $0.1 \mathrm{MPa}$ [20]. For testing samples under different incident pressures, the test duration $t$ was set to $1 \mathrm{~min}$.

\subsection{Forming Device}

The forming device shown in Figure 4 comprised a locking block, mask, seal ring, workpiece, and micromold. First, the TA2 titanium foil was placed on the top surface of the mold to cover the mold openings completely. Next, the mask was placed on the TA2 titanium foil, and the mask and micromold were aligned. Subsequently, a locking block was utilized to prevent the foil material from flowing into the micromold cavity and wrinkling in the blank holding area by exerting a blank holding force of $36 \mathrm{~N}$ on the blank holding area. Hence, the foil material in the blank holding area was undeformed, and the $2 \mathrm{~A} 12$ aluminum foil underwent 


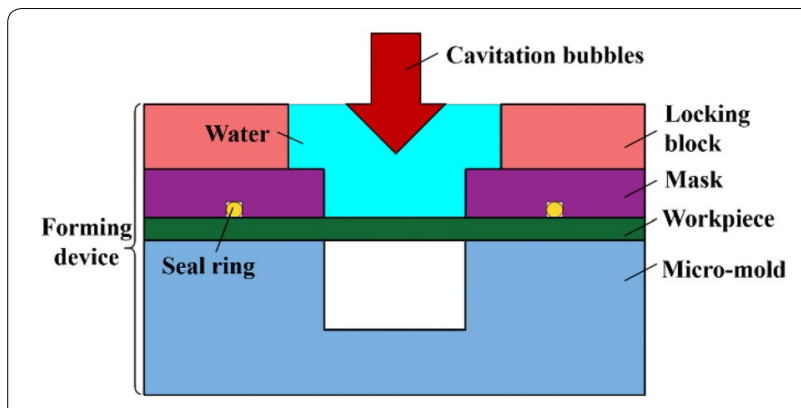

Figure 4 Schematic illustration of forming device

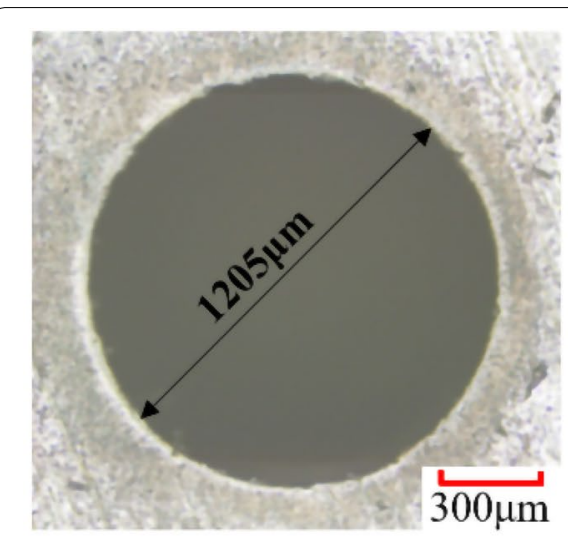

a Vertical view of micromold

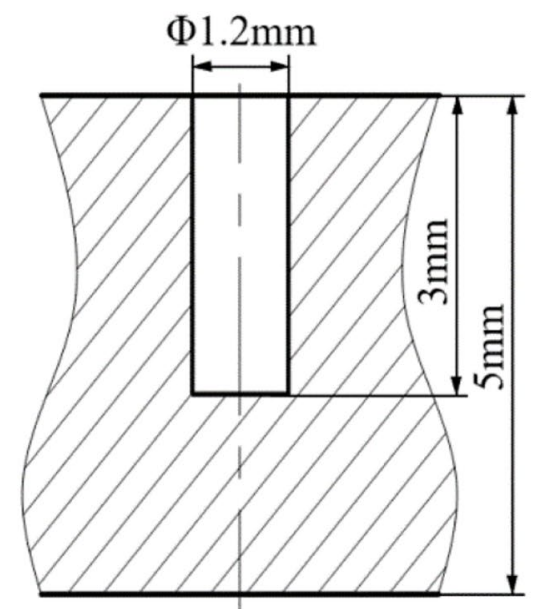

b Structure and size diagram of micromold Figure 5 Structure and size diagram of micromold bulging. Finally, a seal ring was placed between the mask and foil to provide an effective sealing.

The designed micromold is shown in Figure 5. It was fabricated via micro-EDM using a 304 stainless steel cylinder and had a diameter of $\Phi 40 \mathrm{~mm}$ and a height of $5 \mathrm{~mm}$. In this micromold, the diameter and depth of the cavity were $\Phi 1.2 \mathrm{~mm}$ and $3 \mathrm{~mm}$, respectively. After the micromold was processed, it was immersed in an anhydrous alcohol solution, followed by ultrasonic vibration cleaning; subsequently, it was measured using a digital microscope (KEYENCE VHX-1000C). In addition, 304 stainless steel foil was selected as the mask material; the contour of the mask measured $\Phi 40 \mathrm{~mm} \times 2 \mathrm{~mm}$, and the diameter of the mask hole was $\Phi 1.2 \mathrm{~mm}$.

Titanium foils (TA2, purity $>99.6 \%$ ) provided by Shenzhen Hongwang Molding Co., Ltd. were selected as the testing foil material. Its compositions and mechanical properties are listed in Tables 1 and 2, respectively. After the titanium foils were cut into square specimens measuring $35 \mathrm{~mm} \times 35 \mathrm{~mm}$, cleaning and drying were conducted to prepare the testing samples.

\subsection{Surface Observation and Quality Characterization 2.3.1 Surface Topography Measurement}

After water-jet cavitation shock microbulging was performed, the bulging profile and forming depth of the bulging parts were characterized using a digital microscope (KEYENCE VHX-1000C) to analyze their forming quality. The surface topography and roughness value of the micromold and TA2 titanium foils were measured using a shape measurement laser microscopy system (VK-X250K) [21].

\subsubsection{Measurement of Thickness Thinning Ratio}

Considering that localized necking is a sign of fracture failure, the thickness distribution of the bulging parts obtained via water-jet cavitation shock microbulging must be analyzed. The specimens that were cut along the middle line of the bulging parts should be mounted in a plastic mold filled with a low-viscosity epoxy resin such that its crosssectional thickness can be measured conveniently using a digital microscope and its thickness distribution can be characterized precisely. After the cross section of the mounted specimens was polished well, their thickness was measured using a digital microscope (KEYENCE VHX$1000 \mathrm{C})$. According to the measured thickness of the forming region at measuring points $t_{i}(i=1,2, \ldots, 13)$ as well as

Table 1 Compositions of TA2 titanium foils

\begin{tabular}{lllllll}
\hline Element & $\mathbf{H}$ & $\mathbf{C}$ & $\mathbf{N}$ & $\mathbf{O}$ & $\mathbf{F e}$ & $\mathbf{T i}$ \\
\hline Mass fraction (\%) & 0.01 & 0.08 & 0.08 & 0.10 & 0.12 & Balance \\
\hline
\end{tabular}


Table 2 Mechanical properties of TA2 titanium foils

\begin{tabular}{llll}
\hline Density $\left(\mathbf{k g} / \mathbf{m}^{\mathbf{3}}\right)$ & $\begin{array}{l}\text { Elastic modulus } \\
(\mathbf{G P a})\end{array}$ & $\begin{array}{l}\text { Dynamic yield } \\
\text { strength }(\mathrm{GPa})\end{array}$ & Poisson ratio $\boldsymbol{v}$ \\
\hline 4430 & 107.9 & 1.345 & 0.342 \\
\hline
\end{tabular}

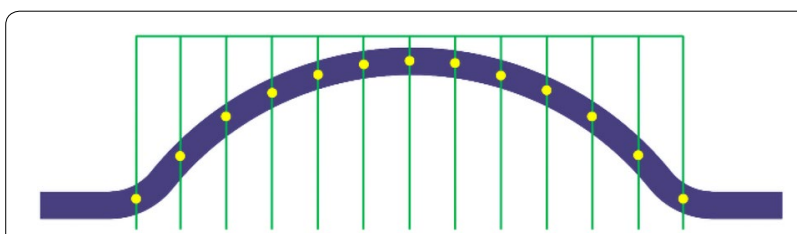

Figure 6 Arrangement of measuring points for measuring thickness distribution of bulging parts good surface quality without evident defects such as mold scratches and cracks was observed. Moreover, wrinkling was not observed in the blank holding area of the bulging part. In addition, the bulging part shown in Figure $7 \mathrm{~b}$ is a spherical cap-shaped sample, indicating that strong plastic deformation occurred and gradually decreased from the center to the periphery. Figure $7 \mathrm{c}$ shows the surface profile of the bulging part. The smooth profile without abrupt change indicates that the foil material flowed into the mold homogeneously during the water-jet cavitation shock microbulging. The maximum forming depth can reach $248.9 \mu \mathrm{m}$ at the center of the bulging part.

The mechanism of microbulging based on water-jet cavitation shock is shown in Figure 8. Cavitation bubbles were generated by injecting a high-speed water jet

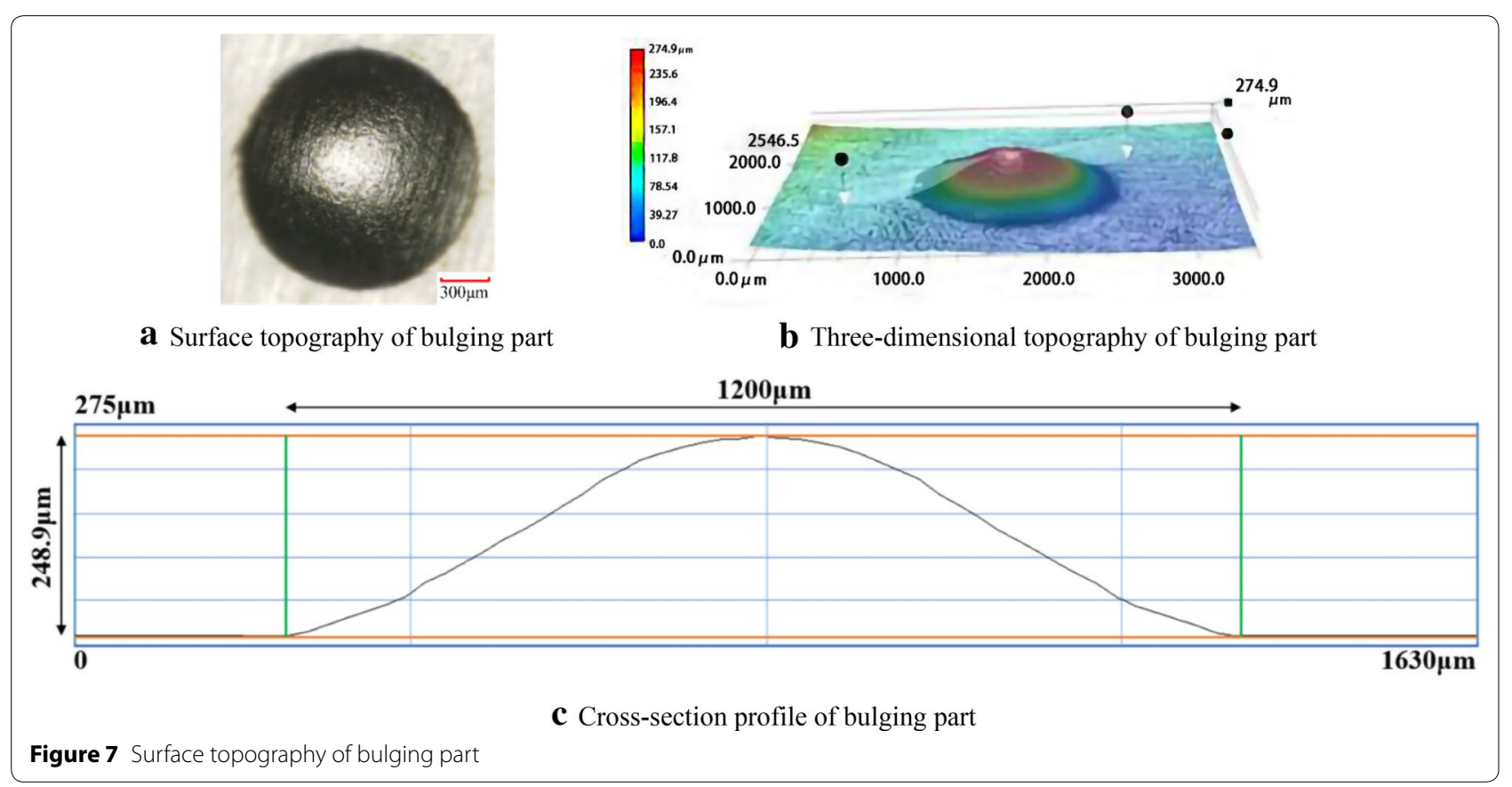

the initial thickness of the TA2 titanium foils $t_{0}$, the thickness thinning ratio $r$ can be calculated using Eq. (2) [22]. The 13 measuring points were arranged as shown in Figure 6 , where the space between two adjacent measurement points was $100( \pm 0.5) \mu \mathrm{m}$.

$$
r=\frac{t_{0}-t_{i}}{t_{0}} \times 100 \%
$$

\section{Results and Discussion}

\subsection{Surface Topography of Bulging Parts}

Figure 7a shows the surface topography of the bulging part under an incident pressure of $20 \mathrm{MPa}$. A relatively

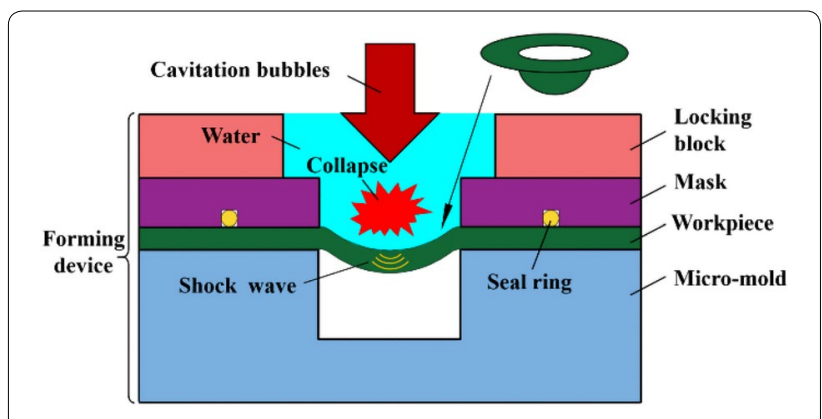

Figure 8 Schematic of water-jet cavitation shock microbulging 
into a water-filled chamber through a nozzle. When the cavitation bubbles approached the surface of the workpiece, a sudden increase in local pressure induced by the significant turbulent pressure pulsation resulted in a concentrated collapse of the cavitation bubbles and hence shock waves [23]. These shock waves formed on the workpiece surface and propagated into the interior of the workpiece owing to the high concentration of energy; consequently, the peak pressure value was in the gigapascal range $[24,25]$. Once the peak value of the collapse pressure exceeded the Hugoniot elastic limit of the foil material, yielding and plastic deformation occurred. The plastic deformation of the workpiece increased until the peak stress was less than the dynamic yield strength of the foil material. Because the loading direction was downward and the workpiece was unconstrained on the bottom side, the foil material flowed downward to fill the mold. The forming mechanism was similar to that of laser-induced cavitation forming, but the forming force was derived from the laser-induced single cavitation bubble instead of the water-jet-induced cluster of cavitation bubbles [26].

\subsection{Forming Depth of Bulging Parts under Different Incident Pressures and Their Profiles}

Table 3 lists the forming depths of the bulging parts under different incident pressures (i.e., $8,12,16$, and 20 $\mathrm{MPa}$ ). For each incident pressure, microbulging tests were performed for eight times, and their average forming depths are listed in Table 3. Figure 9a presents the average surface profile of the bulging parts with different incident pressures. As shown, the forming depth increased with the incident pressure, and its peak value was always located at the center of the bulging part because the cavitation nozzle generated more cavitation bubbles and provided a higher impact load as the incident pressure increased [27]. When the cavitation

Table 3 Forming depth of bulging parts under different incident pressures

\begin{tabular}{llllll}
\hline Incident pressures (MPa) & $\mathbf{8}$ & $\mathbf{1 2}$ & $\mathbf{1 6}$ & $\mathbf{2 0}$ \\
\hline Forming depth $(\mu \mathrm{m})$ & 1 & 79.5 & 129.7 & 144.6 & 230.4 \\
& 2 & 94.1 & 158.5 & 165.7 & 192.3 \\
3 & 57.9 & 137.0 & 154.6 & 208.5 \\
4 & 89.1 & 132.7 & 190.3 & 234.0 \\
5 & 78.5 & 161.2 & 163.9 & 214.5 \\
6 & 93.2 & 140.4 & 146.7 & 248.9 \\
& 7 & 81.9 & 121.2 & 187.2 & 194.7 \\
& 8 & 66.8 & 115.7 & 148.8 & 218.1 \\
& Average & 80.1 & 137.1 & 162.7 & 217.7 \\
\hline
\end{tabular}

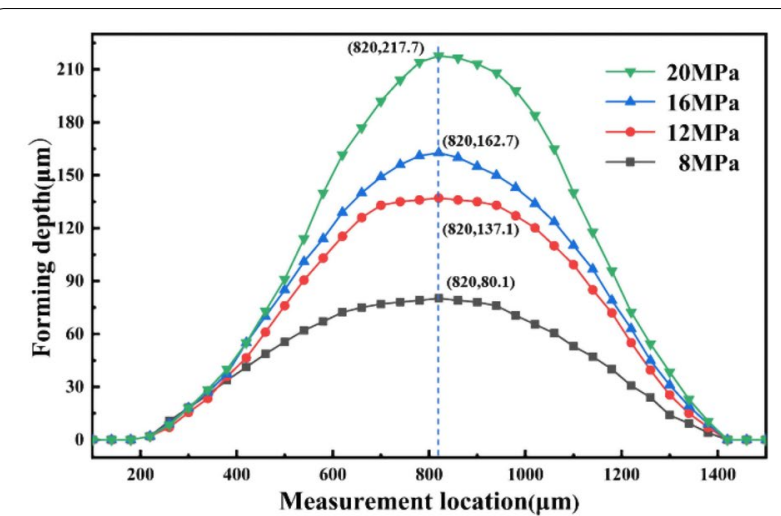

a Average profile of bulging parts under different incident pressures

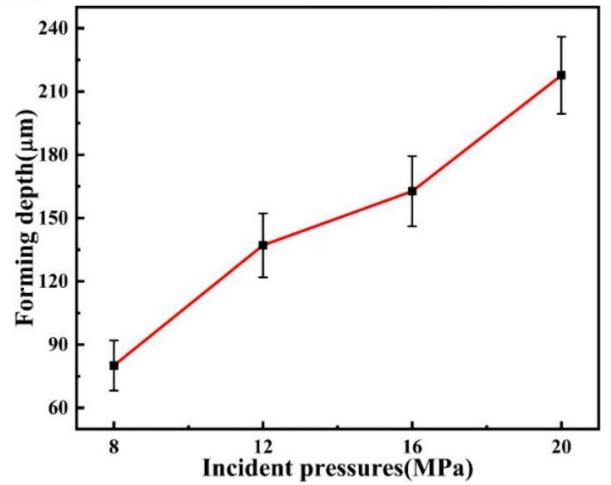

b Average forming depth vs. incident pressure

Figure 9 Average profile and forming depth of bulging parts under different incident pressures

bubbles approached the surface of the workpiece, their sudden collapse induced a higher shock wave pressure, and they propagated into the workpiece such that the strain energy in the workpiece as well as the forming depth increased. Figure $9 \mathrm{~b}$ shows the change curve of the average forming depth with incident pressure, from which it can be observed that the plastic deformation of the TA2 titanium foil increased nonlinearly with the incident pressure. More specifically, as the incident pressure increased from 8 to $16 \mathrm{MPa}$, the forming depth improved at a decreasing rate. As the incident pressure increased from 16 to $20 \mathrm{MPa}$, the forming depth increased significantly. This can be attributed to the following reasons: (1) The air enclosed in the micromold cavity during water-jet cavitation shock microbulging was compressed gradually because it could not be expelled successfully, as the flow resistance of the foil material increased significantly; (2) The dynamic yield limit of the foil material during waterjet cavitation shock microbulging increased continually owing to the hardened effect and hence a larger force was required for a further deformation; (3) As the water-jet cavitation shock microbulging was conducted under a 
standoff distance $S=120 \mathrm{~mm}$ and an incident pressure less than $16 \mathrm{MPa}$, most of the cavitation bubbles collapsed before reaching the workpiece surface; therefore, the shock energy was relatively low and the improvement in forming depth was insignificant [18]. However, as the incident pressure increased to $20 \mathrm{MPa}$, the cavitation bubbles enlarged. They collapsed on the workpiece surface; hence, a greater shock energy was generated and the forming depth increased significantly. Furthermore, as shown in Figure 9a, the peak of most curves for different incident pressures was located near the central axis of the forming parts, as indicated by the dashed blue line, except for the curve under an incident pressure of 20 $\mathrm{MPa}$. The peak of the curve under the incident pressure of $20 \mathrm{MPa}$ shifted slightly toward the right. This phenomenon was likely induced by some uncertain experimental factors or measurement errors, which will be investigated in detail in future studies.

\subsection{Surface Morphology of Bulging Parts}

For the bulging parts formed by the water-jet cavitation shock microbulging process, their surface roughness on the center area was between 1.579 and $1.922 \mu \mathrm{m}$ under an incident pressure of $20 \mathrm{MPa}$, and the difference was moderate. The surface morphology of the micromold, original sample, and bottom area of the bulging parts under different incident pressures are presented in Figure 10a, b, and $\mathrm{c}-\mathrm{f}$, respectively. As shown in Figure 10c-f, no cracks appeared on the bulging parts; however, surface rolling marks were observed at low incident pressures, and they vanished gradually as the incident pressure increased. When the incident pressure reached $20 \mathrm{MPa}$, a surface morphology resembling orange peel waves appeared.

To assess the surface roughness of the bulging parts using a shape measurement laser microscopy system (VK-X250K), the bottom portion of the formed parts was selected as the measurement region because it was difficult to focus on the side of the forming area for the larger forming depth. Subsequently, the sample surface was scanned along three lines denoted as "a" "b" and "c" to obtain the roughness value ( $\mathrm{Ra})$, as shown in Figure 11a. Figure 11b shows the Ra of line "a" within the length of $H=100 \mu \mathrm{m}$ and the corresponding roughness profile. The measured surface roughnesses are summarized in Table 4 in addition to the Ra values of the original sample $(0.288 \mu \mathrm{m})$ and micromold $(0.934 \mu \mathrm{m})$.

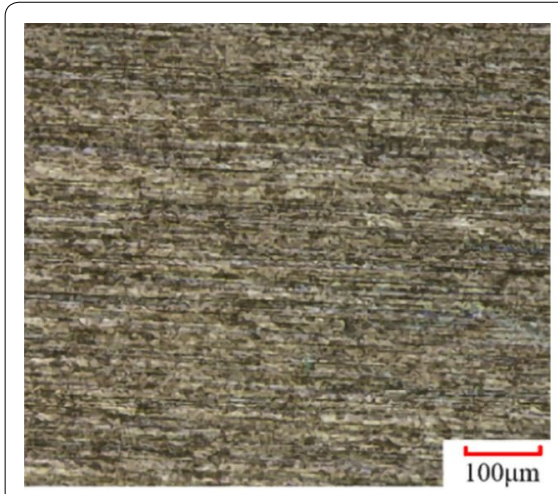

a Micromold

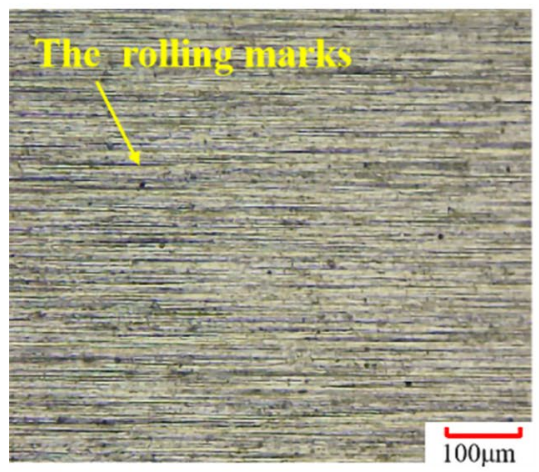

b Original sample

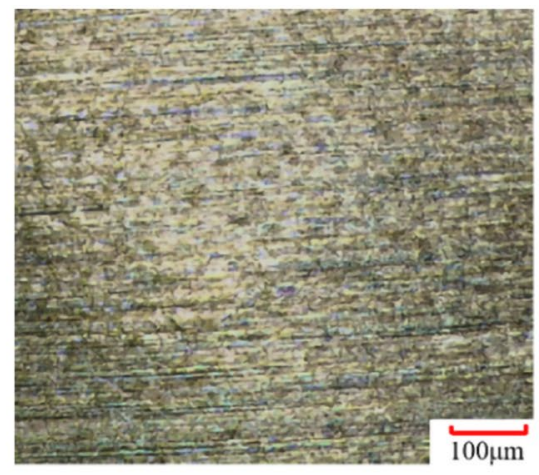

c Bulging parts: $8 \mathrm{MPa}$

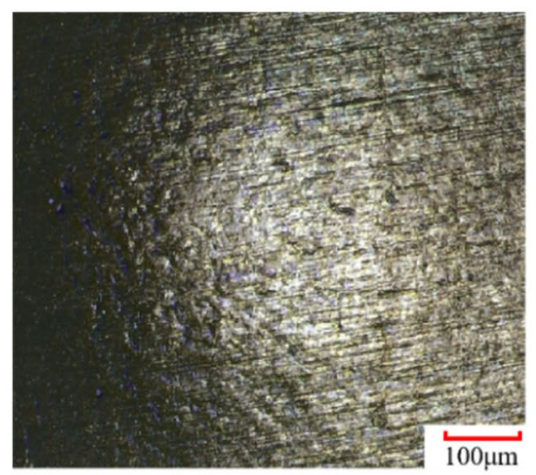

d Bulging parts: $12 \mathrm{MPa}$

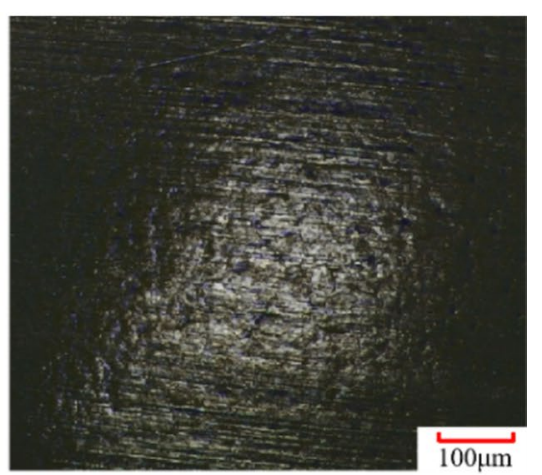

e Bulging parts: $16 \mathrm{MPa}$

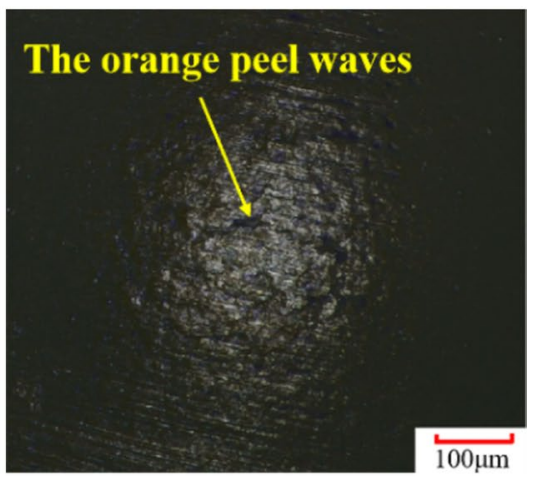

f Bulging parts: $20 \mathrm{MPa}$

Figure 10 Surface morphology of micromold and samples 


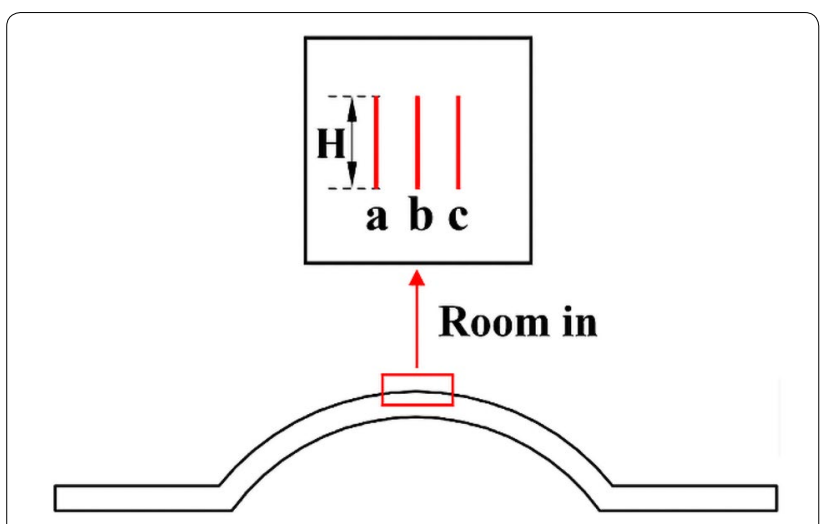

a Measurement regions of surface roughness

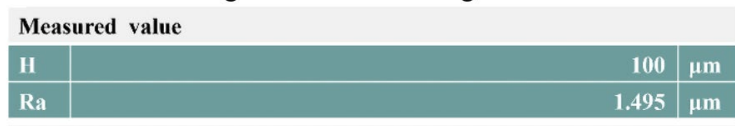

Roughness profile

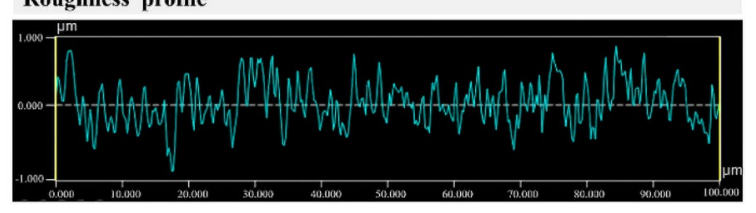

b Surface roughness of line "a"

Figure 11 Measurement of surface roughness for bulging part

To better understand the effect of the incident pressure on the surface quality of the bulging parts, Figure 12 shows the average surface roughness value of the center area of the bulging parts under different incident pressures. As shown, the Ra value of the bulging parts increased with the incident pressure within the range of 8 to $20 \mathrm{MPa}$. This might be attributed to the following reasons: (1) As the incident pressure increased, the mechanical pressure exerted on the workpiece surface increased and caused the foil material to generate a larger plastic deformation by forcing it to flow into the cavity of the rigid micromold. Therefore, a larger friction force and hence a larger surface roughness value of the workpiece were obtained. (2) The shock waves generated by the water-jet cavitation shock microbulging formed micropits on the workpiece surface [28]. The depth of these micropits increased with the incident pressure such that the surface roughness of the workpiece increased significantly. (3) For the surface grains with a lower constraint, the strain incompatibility between neighboring grains was more significant at large deformations; hence, they tended to move or rotate owing to the roughening of intergrains [29].

\subsection{Thickness Thinning Ratio}

The thickness measurement of the bulging parts is illustrated in Figure 13. It was evident that the thinning of the thickness occurred in the entire bulging region. This is because the foil material during forming was subjected to stress stretching and became thinner under the clamping force from the locking block and micromold, the pressure from the micro-mold corner at the entrance, and the flow resistance. In particular, under an incident pressure of 20 $\mathrm{MPa}$, the maximum thickness thinning ratio exceeded $16 \%$ in the fillet region (i.e., locations 1 and 13).

The effect of the incident pressure on the thickness thinning ratio is shown in Figure 14. As shown, the maximum thickness thinning ratio appeared in the fillet region of the bulging part under different incident pressures. Some fluctuations were observed, such as the 10th point under the $16 \mathrm{MPa}$ incident pressure and the 6th point under the $20 \mathrm{MPa}$ incident pressure. They were likely induced by the turbulent pressure pulsation of the highpressure pump. Moreover, the thinning ratio of the bulging parts under different incident pressures decreased from the fillet region to the transition region. This is because the foil material far away from the fillet region was constrained by the micromold and had a lower stress value. However, an irregular thickness distribution was observed from the transition region to the bottom region of the bulging parts. This can be attributed to the inhomogeneous deformation induced by the randomness of the cavitation bubble size and their distributions. When

Table 4 Summary of roughness measurement results

\begin{tabular}{lllllll}
\hline Type & $\begin{array}{l}\text { Incident pressure } \\
\text { (MPa) }\end{array}$ & Forming depth $(\mu \mathrm{m})$ & $\mathbf{R a}(\mu \mathrm{m})$ & & & \\
\cline { 5 - 7 } & & & Line $\mathbf{a}$ & Line $\mathbf{b}$ & Line c & Average \\
\hline Original sample & $\sim$ & $\sim$ & 0.292 & 0.284 & 0.287 & 0.288 \\
Mold & $\sim$ & $\sim$ & 1.042 & 0.814 & 0.947 & 0.934 \\
Bulging parts & 8 & 79.5 & 0.764 & 0.565 & 0.616 & 0.648 \\
& 12 & 137.0 & 0.826 & 1.049 & 0.900 & 0.925 \\
& 16 & 163.9 & 1.219 & 1.358 & 1.201 & 1.259 \\
& 20 & 218.1 & 1.917 & 1.922 & 1.579 & 1.806 \\
\hline
\end{tabular}




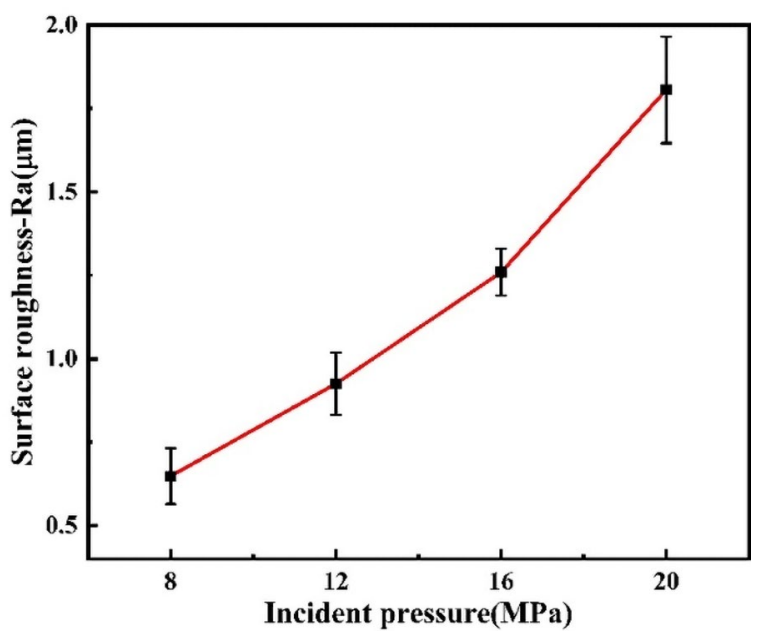

Figure 12 Surface roughness of center area of bulging parts under different incident pressures

the incident pressure was $20 \mathrm{MPa}$, the thinning ratio of the bulging parts increased gradually from the transition region to the bottom region. This is because the cavitation bubbles under this incident pressure can enlarge before collapsing, such that a greater impact load is achieved as the cavitation bubbles collapsed [18]. In this case, the compressive stress exerted on the center of the bulging parts was more pronounced as a result of the significant thinning of the bottom region.

Furthermore, according to the results in Section 3.2, under incident pressures of $8,12,16$ and $20 \mathrm{MPa}$, the corresponding average forming depths were 80.1, 137.1, 162.7 , and $217.7 \mu \mathrm{m}$, respectively, whereas the corresponding maximum thickness thinning ratios were $10.8 \%, 12.9 \%, 14.62 \%$, and $16.76 \%$, respectively. This indicates that the higher the incident pressure, the stronger is the shock wave induced by the collapse of the cavitation bubbles. Hence, the deformation was deeper and the thinning ratio was larger under the incident pressure of $20 \mathrm{MPa}$ than those under other incident pressures. In other words, as the incident pressure increased from 8 $\mathrm{MPa}$ to $20 \mathrm{MPa}$, the maximum thickness thinning ratio of the formed components increased from $10.8 \%$ to $16.76 \%$. It is noteworthy that the thickness thinning ratio was below $10 \%$ for most of the bulging parts except their fillet regions. Consequently, the uniformity of the waterjet cavitation shock microbulging parts was significantly better than those of the laser shock microforming parts because the spatial profile of the laser beam was nonuniform over the entire area of the spot and the shock wave pressure obeyed the Gaussian spatial distribution $[30,31]$. This implies that the water-jet cavitation shock microbulging process can provide a more uniform thickness distribution owing to the relatively uniform force.

Figure 15 presents the force exerted on the metal foil during the water-jet cavitation shock microbulging. Because it was difficult for the partial foil material to be clamped firmly between the locking block and the

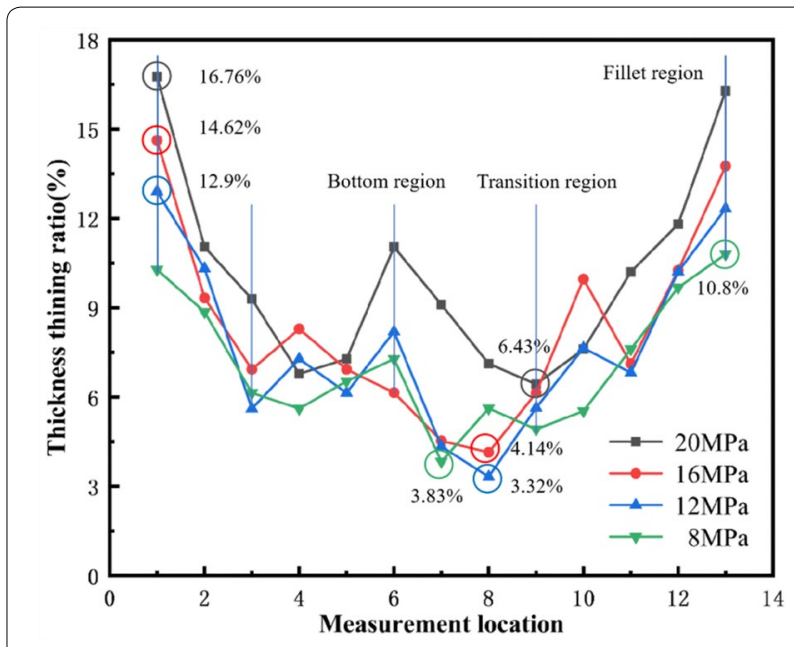

Figure 14 Distribution of thickness thinning ratio under different incident pressures

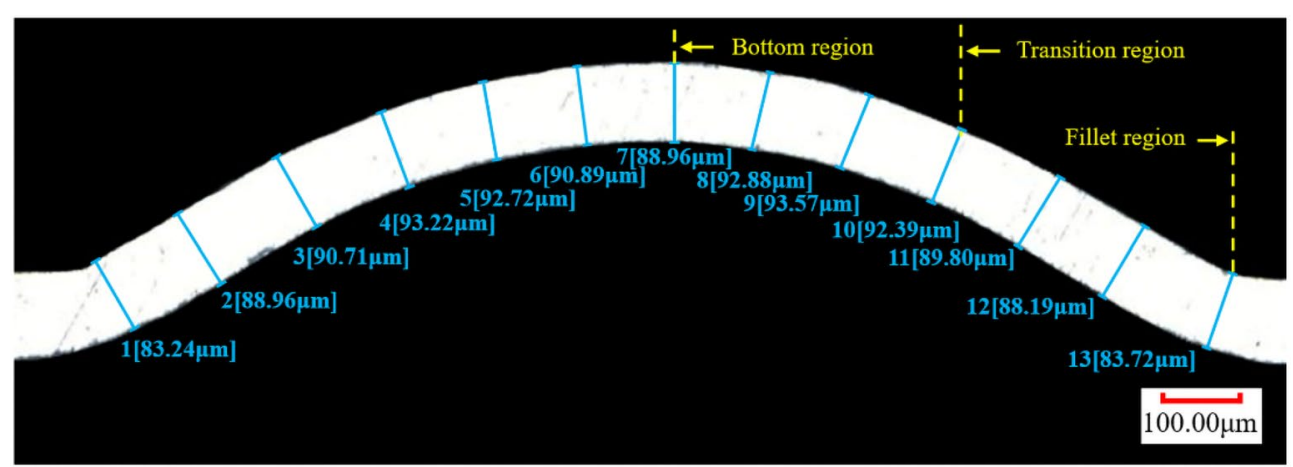

Figure 13 Thickness measurement of bulging parts 


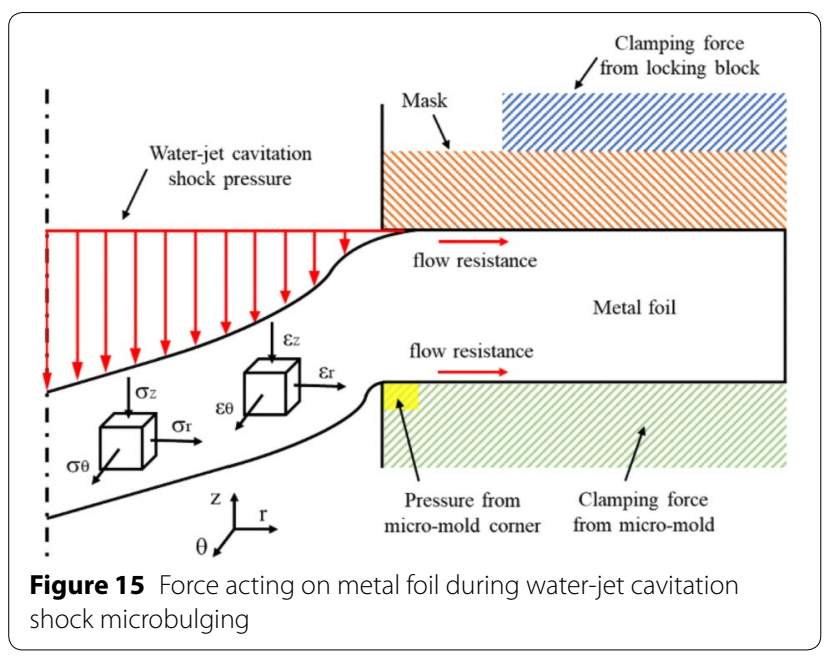

micromold surface, workpiece forming was realized via a reduction in thickness and an elongation along the inplane direction. This resulted in a decrease in the overall thickness of the bulging parts. Moreover, the foil material located in the fillet region assumed the pressure from the corner of the micromold, thereby generating an extensive plastic deformation. In addition, the maximum thickness thinning ratio tended to appear in this region. Accordingly, it can be inferred that cracking will occur in this region if the incident pressure is extremely high.

\section{Conclusions}

A novel water-jet cavitation shock microbulging process was proposed, and the effect of the incident pressure on the forming depth and profile of the bugling parts was investigated in addition to their surface topography and thickness distribution. The main conclusions are as follows.

(1) The water-jet cavitation shock microbulging process can be used for the plastic forming of TA2 titanium foil successfully.

(2) The incident pressure is an important parameter in the process of water-jet cavitation shock microbulging. The plastic deformation of TA2 titanium foil increased nonlinearly with the incident pressure.

(3) A spherical cap can be obtained using water-jet cavitation shock microbulging. When the incident pressure was $20 \mathrm{MPa}$, the height of the cap exceeded $240 \mu \mathrm{m}$.

(4) As the incident pressure increased, the surface roughness of the center area of the bulging parts increased gradually.

(5) The thickness thinning ratio was generally below $10 \%$ for most of the bulging parts except their fil- let regions. This indicates that the forming area of the bulging parts exhibited a considerably uniform thickness distribution.

\section{Acknowledgements}

The authors sincerely thank Professor Li of Jiangsu University for his critical discussion and reading during manuscript preparation.

\section{Authors' Contributions}

FL and HF were in charge of the whole trial; FL and YW wrote the manuscript; and other authors assisted with sampling and laboratory analyses. All authors have read and approved the final manuscript.

\section{Authors' Information}

Fuzhu Li born in 1971, is currently an associate professor at Jiangsu University, China. He received his PhD degree from Jiangsu University, China, in 2009. His research interests include mechanical electro-hydraulic systems and cavitation of hydraulic systems.

\section{Haiyang Fan born in 1994, is currently a master candidate at the School of} Mechanical Engineering, Jiangsu University, China.

Yun Wang born in 1975, is currently a professor at Jiangsu University, China. He received his PhD degree from Yanshan University, China, in 2003. His research interests include laser micromachining, surface engineering, and non-traditional machining processes.

\section{Funding}

Supported by National Natural Science Foundation of China (Grant Nos. 51575245, 51679112), Jiangsu Province Key Research and Development Program of China (Grant No. BE2016161), Jiangsu Province "Six Talents Peak" Project of China (Grant No. XNYQC-002).

\section{Competing Interests}

The authors declare no competing financial interests.

Received: 2 January 2020 Revised: 10 November 2020 Accepted: 18 November 2020

Published online: 05 January 2021

\section{References}

[1] U Engel, R Eckstein. Microforming-from basic research to its realization. Journal of Materials Processing Technology, 2002, 125(9): 35-44.

[2] Q J Zhao, C J Wang, H P Yu, et al. Micro bulging of thin T2 copper sheet by electromagnetic forming. Transactions of Nonferrous Metals Society of China, 2011, 21(2): 461-464.

[3] J G Liu, M W Fu, J Lu, et al. Influence of size effect on the spring back of sheet metal foils in micro-bending. Computational Materials Science, 2011, 50(9): 2604-2614.

[4] D B Shan, J Xu, C J Wang, et al. The state of the art in plastic micro-forming. Materials China, 2016, 35(4): 251-261.

[5] M Geiger, U Engel. Micro-forming a challenge to the plasticity research community-addressed to the 40th anniversary of the JSTP. Journal of the JSTP, 2002, 494(43): 171-172.

[6] O Dan, H Soyama. Cavitation shotless peening for improvement of fatigue strength of carbonized steel. International Journal of Fatigue, 2003, 25(9): 1217-1222

[7] A K Lidtke, V F Humphrey, S R Turnock. Feasibility study into a computational approach for marine propeller noise and cavitation modelling. Ocean Engineering, 2016, 120(1): 152-159.

[8] H L Man, S L Kil, C P Won, et al. On the synthesis of an underwater ship hull cleaning robot system. International Journal of Precision Engineering and Manufacturing, 2012, 13(11): 1965-1973.

[9] Y Q Tao, J Cai, X L Huai, et al. Experimental study on degradation of organic wastewater based on high pressure cavitating jet impingement. Journal of Engineering Thermo Physics, 2018, 39(12): 2738-2744. 
[10] R H Wang, C H Wan, W D Zhao, et al. Mechanism of heavy oil viscosity reduction by cavitation jet. Journal of China University of Petroleum, 2019 43(5): 101-108

[11] H Soyama. Cavitation peening: A review. Metals, 2020, 10(2): 1-27.

[12] A Marcon, S N Melkote, J Castle, et al. Effect of jet velocity in co-flow water cavitation jet peening. Wear, 2016, 360(8): 38-50.

[13] H Tsuda, DY Ju, T Uchiyama, et al. Residual stress in a quenched gear shaft treated by water cavitation peening. Materials Science Forum, 2005, 490-491: 364-369.

[14] H Soyama, K Saito. Peen forming of duralumin plate by using a cavitating jet in air. Proceedings of the 7th Pacific Rim International Conference on Water Jetting Technology, 2003: 429-436.

[15] H Soyama. Effect of nozzle geometry on a standard cavitation erosion test using a cavitating jet. Wear, 2012, 297(1): 895-902.

[16] H Soyama. Enhancing the aggressive intensity of a cavitating jet by means of the nozzle outlet geometry. Journal of Fluids Engineering, 2011, 133(10): 101-301

[17] F Z Li, Z R Tan, L Y Chen, et al. Study on dynamic evolution of cavitation clouds and optimization of standoff distance in water cavitation peening. Journal of Mechanical Engineering, 2019, 55(9): 120-126.

[18] Y Yamauchi, H Soyama, Y Adachi, et al. The suitable region of high-speed submerged water-jets for cutting and peening. JSME International Journal Series $B, 1995,38(1): 31-38$.

[19] H Soyama, K Nagasaka, O Takakuwa, et al. Optimum injection pressure of a cavitating jet on introduction of compressive residual stress into stainless steel. Journal of Power and Energy Systems, 2012, 6(2): 63-75.

[20] X Wang, T Qiu, Z Shen, et al. Forming properties of a micro- scale laser dynamic flexible forming technique. Advanced Manufacturing Processes, 2016, 31(6): 1671-1679.

[21] W Zhang, H Liu, Z Shen, et al. Experimental investigation on the formation behavior for three-layer metal sheets under laser high speed flexible micro-forming. International Journal of Advanced Manufacturing Technology, 2017, 9(1): 3149-3157.
[22] H Soyama, Y Yamauchi, Y Adachi, et al. High-speed observations of the cavitation cloud around a high-speed submerged water-jet. JSME International Journal Series B-Fluids and Thermal Engineering, 1995, 3(8): 245-251.

[23] K Sato, S Ohjimi, Y Sugimoto. Collapsing and impulsive behavior of cavitation clouds on cavitating water-jet impinging on solid wall. JSME International Journal Series B, 2009, 7(50): 47-56.

[24] S Fujikawa, T Akamatsu. Experimental investigations of cavita- tion bubble collapse by a water shock tube. JSME International Journal Series B, 1978, 21(152): 223-230

[25] T N Chen, Z N Guo, B W Zeng, et al. Experimental research and numerical simulation of the punch forming of aluminum foil based on a laserinduced cavitation bubble. The International Journal of Advanced Manufacturing Technology, 2017, 15(6): 3275-3284.

[26] S Hattori, T Hirose, K Sugiyama. Prediction of cavitation erosion based on the measurement of bubble collapse impact loads. Wear, 2009, 269(7): 507-514.

[27] KW Peng, S C Tian, G S Li, et al. Mapping cavitation impact field in a submerged cavitating jet. Wear, 2018, 15(11): 12-23.

[28] W H Zhang, HX Liu, Z B Shen, et al. Experimental investigation on the formation behavior for three-layer metal sheets under laser high speed flexible micro-forming. The International Journal of Advanced Manufacturing Technology, 2017, 6(13): 3149-3157.

[29] X Wang, Y Ma, Z Shen, et al. Size effects on formability in microscale laser dynamic forming of copper foil. Journal of Materials Processing Technology, 2015, 2(20): 173-183.

[30] C Zheng, S Sun, Z Ji, et al. Numerical simulation and experimentation of micro scale laser bulge forming. International Journal of Machine Tools and Manufacture, 2010, 12(50): 1048-1056.

[31] HX Liu, Z B Shen, X Wang, et al. Experimental and numerical simulation investigation on laser flexible shock micro-bulging. Metals, 2017, 7(93): $1-16$.

\section{Submit your manuscript to a SpringerOpen ${ }^{\odot}$ journal and benefit from:}

- Convenient online submission

- Rigorous peer review

Open access: articles freely available online

- High visibility within the field

- Retaining the copyright to your article

Submit your next manuscript at $\mathbf{s p r i n g e r o p e n . c o m ~}$ 